\title{
Formulation of equations of motion for a simply supported bridge under a moving railway freight vehicle
}

\author{
Ping Lou* and Qing-yuan Zeng \\ School of Civil Engineering \& Architecture, Railway Campus, Central South University, 22 Shao-shan-nan Road, \\ Changsha, Hunan 410075, P.R. China
}

Received 15 July 2004

Accepted 21 September 2006

\begin{abstract}
Based on energy approach, the equations of motion in matrix form for the railway freight vehicle-bridge interaction system are derived, in which the dynamic contact forces between vehicle and bridge are considered as internal forces. The freight vehicle is modelled as a multi-rigid-body system, which comprises one car body, two bogie frames and four wheelsets. The bogie frame is linked with the car body through spring-dashpot suspension systems, and the bogie frame is rigidly linked with wheelsets. The bridge deck, together with railway track resting on bridge, is modelled as a simply supported Bernoulli-Euler beam and its deflection is described by superimposing modes. The direct time integration method is applied to obtain the dynamic response of the vehicle-bridge interaction system at each time step. A computer program has been developed for analyzing this system. The correctness of the proposed procedure is confirmed by one numerical example. The effect of different beam mode numbers and various surface irregularities of beam on the dynamic responses of the vehicle-bridge interaction system are investigated.
\end{abstract}

Keywords: Equation of motion, vehicle-bridge interaction, railway freight vehicle, simply supported bridge, Bernoulli-Euler beam

\section{Introduction}

The dynamic response of bridge structures subjected to moving vehicles has long been an interesting topic in the field of civil engineering. In analyzing this problem, the key step is to derive the equations of motion for the interaction system between moving vehicles and bridge structures. Just as Clough and Penzien [5] presented in their excellent monograph that the formulation of the equations of motion of a dynamic system is possibly the most important, and sometimes the most difficult, phase of the entire analysis procedure.

Some researchers, such as Olsson [11], Lin and Trethewey [8], and Au et al. [1,2], regarded the vehicle-bridge interaction system as two subsystems, that is, vehicle subsystem and bridge subsystem, and it is interaction forces existing at the contact points between the vehicle and the bridge that make the two subsystems coupled, and then derived the equations of motion for this interaction system. The derivation procedures in abovementioned references $[1,2,8,11]$ for the vehicle-bridge interaction system were as follows. First, the equations of motion for the beam element representing the bridge and the vehicles are separately established. Then, the combined equations of motion for the vehicle-bridge interaction element can be derived by eliminating the degrees of freedom (DOFs), which are associated with the parts of the vehicles in direct contact with the beam element, by means of the constraint conditions at the contact points of displacements, velocities and accelerations. Lastly, by assembling the stiffness

\footnotetext{
${ }^{*}$ Corresponding author. E-mail: pinglou@ mail.csu.edu.cn or pingloucsu@126.com.
} 
matrices, the damping matrices, the mass matrices and the vectors of nodal loads of all elements, the global equations of motion for this system were obtained.

Yang and Lin [16] formulated the equations of motion for vehicle-bridge interaction system with one-foot vehicle model using the modified dynamic condensation method [12], with all DOFs associated with the car bodies being condensed on the element level. Later, to simulate the pitching effect of the car body of vehicle, Yang et al. [15] presented a vehicle-bridge interaction element considering vehicle's pitching effect and then the equations of motion for vehicle-bridge interaction system with two-foot vehicle model were established by using the modified dynamic condensation method [12].

Frýba [6] formulated, respectively, in his excellent monograph, the differential equations of motion of moving one-axle, two-axle and multi-axle mass-spring-damper vehicles and beam using the d'Alembert principle.

Other researchers regarded the vehicle-bridge interaction system as an entire system and directly derived the equations of motion for this interaction system, but they modelled the vehicles differently and used different methods. Based on the Lagrangian equation [5], Wen [14] directly derived the equations of motion for the vehicle bridge interaction system, in which the vehicle is modelled as a two-axle mass-spring system having 4 DOFs, and Humar and Kashif [7] directly set up the equations of motion for this interaction system, in which the vehicle is modelled as a one-axle mass-spring-damper system possessing 2 DOFs. Recently, based on the Hamilton principle, Lou and Zeng [9] directly derived the equations of motion for this interaction system, in which the vehicle is modelled as a four-wheelset 10 DOFs mass-spring-damper system with two-stage suspension systems.

In the references $[1,2,9]$, the vehicle is modelled as a 10 DOFs four-wheelset mass-spring-damper system with two-stage suspension systems. To the authors' knowledge, however, the equations of motion for the vehicle-bridge interaction system with a 10 DOFs four-wheelset railway freight vehicle having one-stage suspension systems did not be presented in the existing literatures.

In this paper, the railway freight vehicle and the bridge are regarded as an entire dynamic system. Based on energy approach, the equations of motion in matrix form for the railway freight vehicle-bridge interaction system are derived, in which the dynamic contact forces between vehicle and bridge are considered as internal forces. The freight vehicle is modelled as a multi-rigid-body system, which comprises one car body, two bogie frames and four wheelsets. The bogie frame is linked with the car body through spring-dashpot suspension systems, and the bogie frame is rigidly linked with wheelsets. The bridge deck, together with railway track resting on bridge, is modelled as a simply supported Bernoulli-Euler beam and its deflection is described by superimposing modes. The equations of motion can be solved by direct time integration method such as Newmark- $\beta$ method [10] or Wilson- $\theta$ method [3], to obtain simultaneously the dynamic responses of vehicle, of contact forces, and of bridge. A computer program has also been developed for analyzing the vehicle-bridge interaction system.

\section{Hamilton's principle}

It is well known that the Hamilton's principle can be expressed in the form [5]

$$
\int_{t_{1}}^{t_{2}} \delta(T-V) \mathrm{d} t+\int_{t_{1}}^{t_{2}} \delta W_{\mathrm{nc}} \mathrm{d} t=0
$$

where, $T$ denotes the kinetic energy for an entire dynamic system, $V$ denotes the potential energy for an entire dynamic system, $\delta W_{\mathrm{nc}}$ denotes the virtual work done by the nonconservative forces for an entire dynamic system, and $\delta$ is the variation symbol. The application of this principle leads directly to the equations of motion for any given system.

\section{Model and formulations}

\subsection{Models of vehicle and bridge}

Figure 1 shows a typical vehicle-bridge interaction system with a four-wheelset railway freight vehicle running on it. It is assumed that each wheelset of vehicle always keeps in contact with the rails. The railway freight vehicle 


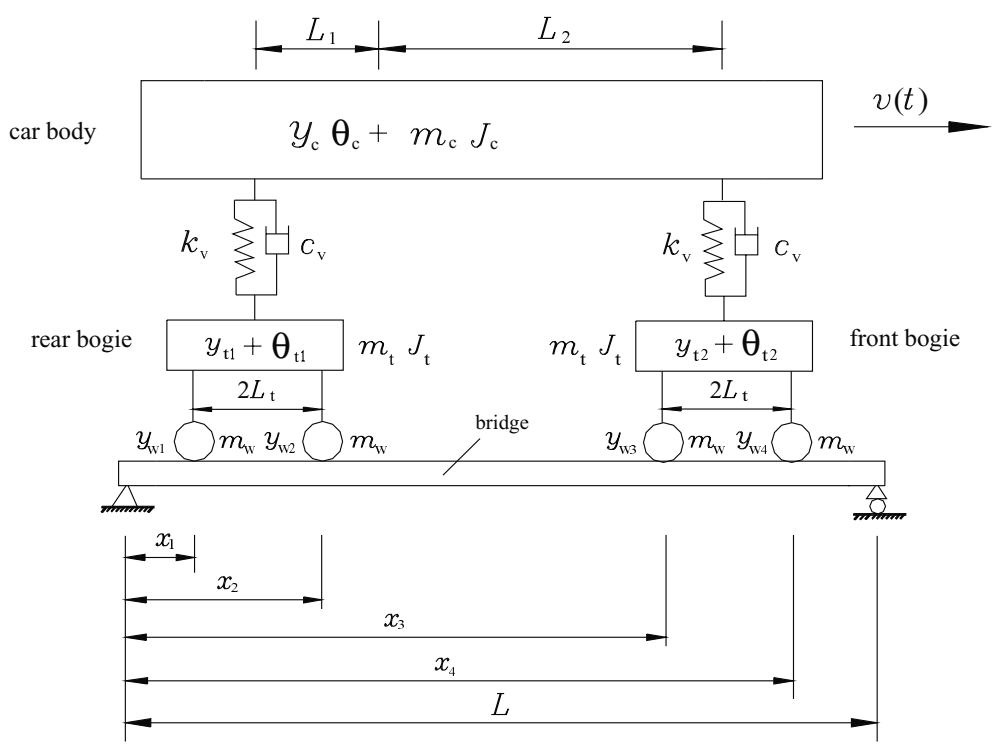

Fig. 1. Model of vehicle-bridge interaction system with a four-wheelset railway freight vehicle.

is modelled as multi-rigid-body system, which comprises one car body, two bogie frames and four wheelsets. The bogie frame is linked with the car body through spring-dashpot suspension systems, and the bogie frame is rigidly linked with wheelsets. The car body is modelled as a rigid body with a mass $m_{\mathrm{c}}$ and a mass moment of inertia $J_{\mathrm{c}}$ about the transverse horizontal axis through its centre of gravity. Similarly, each bogie frame is considered as a rigid body having a mass $m_{\mathrm{t}}$ and a mass moment of inertia $J_{\mathrm{t}}$ about the transverse horizontal axis through its centre of gravity. Each wheelset has a mass $m_{\mathrm{w}}$. The spring and shock absorber in the suspension system between each bogie frame and car body are characterized by spring stiffness $k_{\mathrm{v}}$ and damping coefficient $c_{\mathrm{v}}$, respectively. As the car body is assumed to be rigid, its motion may be described by the vertical displacement $y_{\mathrm{c}}$ and rotation $\theta_{\mathrm{c}}$ at its centre of gravity. Similarly, the motions of the rear bogie frame may be described by the vertical displacement $y_{\mathrm{t} 1}$ and rotation $\theta_{\mathrm{t} 1}$ at its centre of gravity; the motions of the front bogie frame may be described by the vertical displacement $y$ t 2 and rotation $\theta_{\mathrm{t} 2}$ at its centre of gravity. The motions of four wheelsets may be described by the vertical displacement $y_{\mathrm{W} 1}, y_{\mathrm{W} 2}, y_{\mathrm{W} 3}$ and $y_{\mathrm{W} 4}$, respectively. Therefore, the total number of DOFs for one freight vehicle is 10 . The vertical displacement of wheelset, however, is constrained by the displacement of bridge, the vertical displacement and rotation of rear bogie frame is constrained by the vertical displacements of the two wheelsets of rear bogie, and the vertical displacement and rotation of front bogie frame is constrained by the vertical displacements of the two wheelsets of front bogie. Consequently, the independent DOFs for one freight vehicle become 2 , that is, $y_{\mathrm{c}}$ and $\theta_{\mathrm{c}}$ are independent DOFs. It is assumed that the downward vertical displacements and clockwise direction rotation of vehicle are taken as positive and that they are measured with reference to their respective static equilibrium positions before coming onto the bridge. The vehicle proceeds with velocity $v(t)$ and acceleration $a(t)$ in the longitudinal direction at time $t$.

The bridge deck, together with the railway track resting on bridge, is modelled as a simply supported uniform Bernoulli-Euler beam, and the mass per unit length of the beam is $\bar{m}_{\mathrm{b}}$.

It is assumed that the downward deflection of beam is taken as positive and that it is measured with reference to its vertical static equilibrium positions. Let $r(x)$ denote the top surface irregularities of beam that are defined as the vertically downward departure from the mean horizontal profile.

\subsection{Constraint equations at the contact points between wheelsets and beam}

Since each wheelset of vehicle is assumed to be always in contact with the beam, the constraint equations at the four contact points between vehicle and beam can then be written as 


$$
\begin{aligned}
y_{\mathrm{W} h}(t)= & {\left.[y(x, t)+r(x)]\right|_{x=x_{h}} \text { for } h=1-4 } \\
\dot{y}_{\mathrm{W} h}(t)= & \mathrm{d} y_{\mathrm{W} h}(t) / \mathrm{d} t=\left.[\partial y(x, t) / \partial t+v \cdot \partial y(x, t) / \partial x+v \cdot \mathrm{d} r / \mathrm{d} x]\right|_{x=x_{h}} \text { for } h=1-4 \\
\ddot{y}_{\mathrm{W} h}(t)= & \mathrm{d}^{2} y_{\mathrm{W} h}(t) / \mathrm{d} t^{2}=\left[\partial^{2} y(x, t) / \partial t^{2}+2 v \cdot \partial^{2} y(x, t) / \partial x \partial t+v^{2} \cdot \partial^{2} y(x, t) / \partial x^{2}\right. \\
& \left.+a \cdot \partial y(x, t) / \partial x+v^{2} \cdot \mathrm{d}^{2} r / \mathrm{d} x^{2}+a \cdot \mathrm{d} r / \mathrm{d} x\right]\left.\right|_{x=x_{h}} \text { for } h=1-4
\end{aligned}
$$

where, as shown in Fig. 1, $x_{1}$ denotes the longitudinal distance between the rear wheelset of rear bogie and the left end point of the beam, $x_{2}$ denotes the longitudinal distance between the front wheelset of rear bogie and the left end point of the beam, $x_{3}$ denotes the longitudinal distance between the rear wheelset of front bogie and the left end point of the beam, $x_{4}$ denotes the longitudinal distance between the front wheelset of front bogie and the left end point of the beam, $y_{\mathrm{w} 1}(t), y_{\mathrm{w} 2}(t), y_{\mathrm{w} 3}(t)$ and $y_{\mathrm{w} 4}(t)$ denote the vertical displacement of the rear wheelset of rear bogie, of the front wheelset of rear bogie, of the rear wheelset of front bogie, and of the front wheelset of front bogie of the vehicle, respectively, $y(x, t)$ denotes the vertical displacement of beam at point $x$ and time $t$, and the dot above symbol denotes differentiation with respect to time $t$.

For a simply supported and prismatic beam, the vertical deflection $y(x, t)$ of beam is given by $[4,13]$

$$
y(x, t)=\sum_{j=1}^{n} A_{j} \sin (j \pi x / L)
$$

where $A_{j}$ denotes modal amplitude of the $j$ th mode, and is a function of time, $j$ denotes the number of half waves in which the vibrating beam is subdivided, $n$ denotes the total number of modes, and $L$ denotes the length of beam.

The differentiation of the function $y(x, t)$ with respective to $x$ and $t$ can be written as

$$
\begin{aligned}
& \frac{\partial y(x, t)}{\partial t}=\sum_{j=1}^{n} \dot{A}_{j} \sin (j \pi x / L) \\
& \frac{\partial y(x, t)}{\partial x}=\sum_{j=1}^{n} A_{j} \cdot \frac{j \pi}{L} \cos (j \pi x / L) \\
& \frac{\partial^{2} y(x, t)}{\partial x^{2}}=-\sum_{j=1}^{n} A_{j} \cdot\left(\frac{j \pi}{L}\right)^{2} \sin (j \pi x / L) \\
& \frac{\partial^{2} y(x, t)}{\partial x \partial t}=\sum_{j=1}^{n} \dot{A}_{j} \cdot \frac{j \pi}{L} \cos (j \pi x / L) \\
& \frac{\partial^{2} y(x, t)}{\partial t^{2}}=\sum_{j=1}^{n} \ddot{A}_{j} \sin (j \pi x / L)
\end{aligned}
$$

Substituting Eqs (5-10) into Eqs (2-4), one obtains

$$
\begin{aligned}
& y_{\mathrm{W} h}(t)=\left.\left[\sum_{j=1}^{n} A_{j} \sin (j \pi x / L)+r(x)\right]\right|_{x=x_{h}} \text { for } h=1-4 \\
& \dot{y}_{\mathrm{W} h}(t)=\left.\left[\sum_{j=1}^{n} \dot{A}_{j} \sin (j \pi x / L)+v \cdot \sum_{j=1}^{n} A_{j} \cdot \frac{j \pi}{L} \cos (j \pi x / L)+v \cdot r^{\prime}(x)\right]\right|_{x=x_{h}} \text { for } h=1-4
\end{aligned}
$$




$$
\begin{aligned}
\ddot{y}_{\mathrm{W} h}(t)= & {\left[\sum_{j=1}^{n} \ddot{A}_{j} \sin (j \pi x / L)+2 v \cdot \sum_{j=1}^{n} \dot{A}_{j} \cdot \frac{j \pi}{L} \cos (j \pi x / L)-v^{2} \cdot \sum_{j=1}^{n} A_{j} \cdot\left(\frac{j \pi}{L}\right)^{2} \sin (j \pi x / L)\right.} \\
& \left.+a \cdot \sum_{j=1}^{n} A_{j} \cdot \frac{j \pi}{L} \cos (j \pi x / L)+v^{2} \cdot r^{\prime \prime}(x)+a \cdot r^{\prime}(x)\right]\left.\right|_{x=x_{h}} \quad \text { for } h=1-4
\end{aligned}
$$

where, the prime denotes differentiation with respect to coordinate $x$.

\subsection{Constraint equations between wheelsets and bogie frames}

Since the bogie frame is rigidly linked with wheelsets, the constraint equations between the rear bogie frame and the two wheelsets of rear bogie can then be written as

$$
\begin{aligned}
& y_{\mathrm{t} 1}(t)=\frac{1}{2}\left[y_{\mathrm{w} 1}(t)+y_{\mathrm{w} 2}(t)\right] \\
& \dot{y}_{\mathrm{t} 1}(t)=\frac{1}{2}\left[\dot{y}_{\mathrm{w} 1}(t)+\dot{y}_{\mathrm{w} 2}(t)\right] \\
& \ddot{y}_{\mathrm{t} 1}(t)=\frac{1}{2}\left[\ddot{y}_{\mathrm{w} 1}(t)+\ddot{y}_{\mathrm{w} 2}(t)\right] \\
& \theta_{\mathrm{t} 1}(t)=\left[y_{\mathrm{W} 2}(t)-y_{\mathrm{w} 1}(t)\right] / 2 L_{\mathrm{t}} \\
& \dot{\theta}_{\mathrm{t} 1}(t)=\left[\dot{y}_{\mathrm{W} 2}(t)-\dot{y}_{\mathrm{w} 1}(t)\right] / 2 L_{\mathrm{t}} \\
& \ddot{\theta}_{\mathrm{t} 1}(t)=\left[\ddot{y}_{\mathrm{w} 2}(t)-\ddot{y}_{\mathrm{W} 1}(t)\right] / 2 L_{\mathrm{t}}
\end{aligned}
$$

where, $L_{\mathrm{t}}$ denotes the half of the bogie wheelbase.

Substituting Eqs (11-13) into Eqs (14-19), one obtains

$$
\begin{aligned}
y_{\mathrm{t}_{1}}(t)= & \frac{1}{2}\left[\sum_{j=1}^{n} A_{j} \sin \left(j \pi x_{1} / L\right)+r\left(x_{1}\right)+\sum_{j=1}^{n} A_{j} \sin \left(j \pi x_{2} / L\right)+r\left(x_{2}\right)\right] \\
\dot{y}_{\mathrm{t} 1}(t)= & \frac{1}{2}\left[\sum_{j=1}^{n} \dot{A}_{j} \sin \left(j \pi x_{1} / L\right)+v \cdot \sum_{j=1}^{n} A_{j} \cdot \frac{j \pi}{L} \cos \left(j \pi x_{1} / L\right)+v \cdot r^{\prime}\left(x_{1}\right)+\right. \\
& \left.\sum_{j=1}^{n} \dot{A}_{j} \sin \left(j \pi x_{2} / L\right)+v \cdot \sum_{j=1}^{n} A_{j} \cdot \frac{j \pi}{L} \cos \left(j \pi x_{2} / L\right)+v \cdot r^{\prime}\left(x_{2}\right)\right] \\
\ddot{y}_{\mathrm{t} 1}(t)= & \frac{1}{2}\left[\sum_{j=1}^{n} \ddot{A}_{j} \sin \left(j \pi x_{1} / L\right)+2 v \cdot \sum_{j=1}^{n} \dot{A}_{j} \cdot \frac{j \pi}{L} \cos \left(j \pi x_{1} / L\right)-v^{2} \cdot \sum_{j=1}^{n} A_{j} \cdot\left(\frac{j \pi}{L}\right)^{2} \sin \left(j \pi x_{1} / L\right)+\right. \\
& a \cdot \sum_{j=1}^{n} A_{j} \cdot \frac{j \pi}{L} \cos \left(j \pi x_{1} / L\right)+v^{2} \cdot r^{\prime \prime}\left(x_{1}\right)+a \cdot r^{\prime}\left(x_{1}\right)+\sum_{j=1}^{n} \ddot{A}_{j} \sin \left(j \pi x_{2} / L\right)+2 v \cdot \sum_{j=1}^{n} \dot{A}_{j} \\
& \cdot \frac{j \pi}{L} \cos ^{n}\left(j \pi x_{2} / L\right)-v^{2} \cdot \sum_{j=1}^{n} A_{j} \cdot\left(\frac{j \pi}{L}\right)^{2} \sin \left(j \pi x_{2} / L\right)+a \cdot \sum_{j=1}^{n} A_{j} \cdot \frac{j \pi}{L} \cos \left(j \pi x_{2} / L\right) \\
& \left.+v^{2} \cdot r^{\prime \prime}\left(x_{2}\right)+a \cdot r^{\prime}\left(x_{2}\right)\right]
\end{aligned}
$$




$$
\begin{aligned}
\theta_{\mathrm{t} 1}(t)= & \frac{1}{2 L_{\mathrm{t}}}\left[\sum_{j=1}^{n} A_{j} \sin \left(j \pi x_{2} / L\right)+r\left(x_{2}\right)-\sum_{j=1}^{n} A_{j} \sin \left(j \pi x_{1} / L\right)-r\left(x_{1}\right)\right] \\
\dot{\theta}_{\mathrm{t} 1}(t)= & \frac{1}{2 L_{\mathrm{t}}}\left[\sum_{j=1}^{n} \dot{A}_{j} \sin \left(j \pi x_{2} / L\right)+v \cdot \sum_{j=1}^{n} A_{j} \cdot \frac{j \pi}{L} \cos \left(j \pi x_{2} / L\right)+v \cdot r^{\prime}\left(x_{2}\right)-\right. \\
& \left.\sum_{j=1}^{n} \dot{A}_{j} \sin \left(j \pi x_{1} / L\right)-v \cdot \sum_{j=1}^{n} A_{j} \cdot \frac{j \pi}{L} \cos \left(j \pi x_{1} / L\right)-v \cdot r^{\prime}\left(x_{1}\right)\right] \\
\ddot{\theta}_{\mathrm{t} 1}(t)= & \frac{1}{2 L_{\mathrm{t}}}\left[\sum_{j=1}^{n} \ddot{A}_{j} \sin \left(j \pi x_{2} / L\right)+2 v \cdot \sum_{j=1}^{n} \dot{A}_{j} \cdot \frac{j \pi}{L} \cos \left(j \pi x_{2} / L\right)-v^{2} \cdot \sum_{j=1}^{n} A_{j} \cdot\left(\frac{j \pi}{L}\right)^{2} \sin \left(j \pi x_{2} / L\right)\right. \\
& +a \cdot \sum_{j=1}^{n} A_{j} \cdot \frac{j \pi}{L} \cos \left(j \pi x_{2} / L\right)+v^{2} \cdot r^{\prime \prime}\left(x_{2}\right)+a \cdot r^{\prime}\left(x_{2}\right)-\sum_{j=1}^{n} \ddot{A}_{j} \sin \left(j \pi x_{1} / L\right) \\
& -2 v \cdot \sum_{j=1}^{n} \dot{A}_{j} \cdot \frac{j \pi}{L} \cos \left(j \pi x_{1} / L\right)+v^{2} \cdot \sum_{j=1}^{n} A_{j} \cdot\left(\frac{j \pi}{L}\right)^{2} \sin \left(j \pi x_{1} / L\right) \\
& \left.-a \cdot \sum_{j=1}^{n} A_{j} \cdot \frac{j \pi}{L} \cos \left(j \pi x_{1} / L\right)-v^{2} \cdot r^{\prime \prime}\left(x_{1}\right)-a \cdot r^{\prime}\left(x_{1}\right)\right]
\end{aligned}
$$

Similarly, one obtains the constraint equations between the front bogie frame and the two wheelsets of front bogie

$$
\begin{aligned}
y_{\mathrm{t} 2}(t)= & \frac{1}{2}\left[\sum_{j=1}^{n} A_{j} \sin \left(j \pi x_{3} / L\right)+r\left(x_{3}\right)+\sum_{j=1}^{n} A_{j} \sin \left(j \pi x_{4} / L\right)+r\left(x_{4}\right)\right] \\
\dot{y}_{\mathrm{t} 2}(t)= & \frac{1}{2}\left[\sum_{j=1}^{n} \dot{A}_{j} \sin \left(j \pi x_{3} / L\right)+v \cdot \sum_{j=1}^{n} A_{j} \cdot \frac{j \pi}{L} \cos \left(j \pi x_{3} / L\right)+v \cdot r^{\prime}\left(x_{3}\right)\right. \\
& \left.+\sum_{j=1}^{n} \dot{A}_{j} \sin \left(j \pi x_{4} / L\right)+v \cdot \sum_{j=1}^{n} A_{j} \cdot \frac{j \pi}{L} \cos \left(j \pi x_{4} / L\right)+v \cdot r^{\prime}\left(x_{4}\right)\right] \\
\ddot{y}_{\mathrm{t} 2}(t)= & \frac{1}{2}\left[\sum_{j=1}^{n} \ddot{A}_{j} \sin \left(j \pi x_{3} / L\right)+2 v \cdot \sum_{j=1}^{n} \dot{A}_{j} \cdot \frac{j \pi}{L} \cos \left(j \pi x_{3} / L\right)-v^{2} \cdot \sum_{j=1}^{n} A_{j} \cdot\left(\frac{j \pi}{L}\right)^{2} \sin \left(j \pi x_{3} / L\right)+\right. \\
& a \cdot \sum_{j=1}^{n} A_{j} \cdot \frac{j \pi}{L} \cos \left(j \pi x_{3} / L\right)+v^{2} \cdot r^{\prime \prime}\left(x_{3}\right)+a \cdot r^{\prime}\left(x_{3}\right)+\sum_{j=1}^{n} \ddot{A}_{j} \sin \left(j \pi x_{4} / L\right) \\
& +2 v \cdot \sum_{j=1}^{n} \dot{A}_{j} \cdot \frac{j \pi}{L} \cos \left(j \pi x_{4} / L\right)-v^{2} \cdot \sum_{j=1}^{n} A_{j} \cdot\left(\frac{j \pi}{L}\right)^{2} \sin \left(j \pi x_{4} / L\right) \\
& \left.+a \cdot \sum_{j=1}^{n} A_{j} \cdot \frac{j \pi}{L} \cos \left(j \pi x_{4} / L\right)+v^{2} \cdot r^{\prime \prime}\left(x_{4}\right)+a \cdot r^{\prime}\left(x_{4}\right)\right]
\end{aligned}
$$




$$
\begin{aligned}
\theta_{\mathrm{t} 2}(t)= & \frac{1}{2 L_{\mathrm{t}}}\left[\sum_{j=1}^{n} A_{j} \sin \left(j \pi x_{4} / L\right)+r\left(x_{4}\right)-\sum_{j=1}^{n} A_{j} \sin \left(j \pi x_{3} / L\right)-r\left(x_{3}\right)\right] \\
\dot{\theta}_{\mathrm{t} 2}(t)= & \frac{1}{2 L_{\mathrm{t}}}\left[\sum_{j=1}^{n} \dot{A}_{j} \sin \left(j \pi x_{4} / L\right)+v \cdot \sum_{j=1}^{n} A_{j} \cdot \frac{j \pi}{L} \cos \left(j \pi x_{4} / L\right)+v \cdot r^{\prime}\left(x_{4}\right)\right. \\
& \left.-\sum_{j=1}^{n} \dot{A}_{j} \sin \left(j \pi x_{3} / L\right)-v \cdot \sum_{j=1}^{n} A_{j} \cdot \frac{j \pi}{L} \cos \left(j \pi x_{3} / L\right)-v \cdot r^{\prime}\left(x_{3}\right)\right] \\
\ddot{\theta}_{\mathrm{t} 2}(t)= & \frac{1}{2 L_{\mathrm{t}}}\left[\sum_{j=1}^{n} \ddot{A}_{j} \sin \left(j \pi x_{4} / L\right)+2 v \cdot \sum_{j=1}^{n} \dot{A}_{j} \cdot \frac{j \pi}{L} \cos \left(j \pi x_{4} / L\right)-v^{2} \cdot \sum_{j=1}^{n} A_{j} \cdot\left(\frac{j \pi}{L}\right)^{2} \sin \left(j \pi x_{4} / L\right)\right. \\
& +a \cdot \sum_{j=1}^{n} A_{j} \cdot \frac{j \pi}{L} \cos \left(j \pi x_{4} / L\right)+v^{2} \cdot r^{\prime \prime}\left(x_{4}\right)+a \cdot r^{\prime}\left(x_{4}\right)-\sum_{j=1}^{n} \ddot{A}_{j} \sin \left(j \pi x_{3} / L\right) \\
& -2 v \cdot \sum_{j=1}^{n} \dot{A}_{j} \cdot \frac{j \pi}{L} \cos \left(j \pi x_{3} / L\right)+v^{2} \cdot \sum_{j=1}^{n} A_{j} \cdot\left(\frac{j \pi}{L}\right)^{2} \sin \left(j \pi x_{3} / L\right) \\
& \left.-a \cdot \sum_{j=1}^{n} A_{j} \cdot \frac{j \pi}{L} \cos \left(j \pi x_{3} / L\right)-v^{2} \cdot r^{\prime \prime}\left(x_{3}\right)-a \cdot r^{\prime}\left(x_{3}\right)\right]
\end{aligned}
$$

\subsection{The total kinetic energy for the vehicle-bridge interaction system}

The total kinetic energy $T$ for the vehicle-bridge interaction system consists of the kinetic energy $T_{\mathrm{c}}$ of the car body due to translation of the centre of gravity and due to rotation about its centre of gravity, the kinetic energy $T_{\mathrm{t}}$ of the two bogie frames masses due to rotation about their individual centre of gravity and due to translation of the centre of gravity considered separately, the kinetic energy $T_{\mathrm{w}}$ of the four wheelsets due to translation of the centre of gravity considered separately, and the kinetic energy $T_{\mathrm{b}}$ of the Bernoulli-Euler beam. It can be expressed as

$$
T=T_{\mathrm{c}}+T_{\mathrm{t}}+T_{\mathrm{w}}+T_{\mathrm{b}}
$$

where

$$
\begin{aligned}
& T_{\mathrm{c}}=\frac{1}{2} m_{\mathrm{c}} \dot{y}_{\mathrm{c}}^{2}+\frac{1}{2} J_{\mathrm{c}} \dot{\theta}_{\mathrm{c}}^{2} \\
& T_{\mathrm{t}}=\frac{1}{2} m_{\mathrm{t}} \dot{y}_{\mathrm{t} 1}^{2}+\frac{1}{2} J_{\mathrm{t}} \dot{\theta}_{\mathrm{t} 1}^{2}+\frac{1}{2} m_{\mathrm{t}} \dot{y}_{\mathrm{t} 2}^{2}+\frac{1}{2} J_{\mathrm{t}} \dot{\theta}_{\mathrm{t} 2}^{2} \\
& T_{\mathrm{w}}=\frac{1}{2} m_{\mathrm{w}}\left(\dot{y}_{\mathrm{W} 1}^{2}+\dot{y}_{\mathrm{W} 2}^{2}+\dot{y}_{\mathrm{W} 3}^{2}+\dot{y}_{\mathrm{W} 4}^{2}\right) \\
& T_{\mathrm{b}}=\frac{1}{2} \int_{0}^{L} \bar{m}_{\mathrm{b}}\left(\frac{\partial y(x, t)}{\partial t}\right)^{2} d x
\end{aligned}
$$

Substituting Eqs (21), (24), (27) and (30) into Eq. (34), Eq. (12) into Eq. (35), Eq. (6) into Eq. (36), then substituting Eqs (33-36) into Eq. (32), one obtains the expression of the total kinetic energy $T$ for the vehicle-bridge interaction system with DOFs of car body and bridge. 


\subsection{The total potential energy for the vehicle-bridge interaction system}

As shown in Fig. 1, the four wheelsets of vehicle run over the bridge, the potential energy of vehicle gravity presents because of the vertical displacement of the bridge. So the total potential energy $V$ for the vehicle-bridge interaction system consists of the spring strain energy $U_{\mathrm{v}}$ of the suspension systems of vehicle between bogie frames and car body, the flexural strain energy $U_{\mathrm{b}}$ of the beam, the potential energy $V_{\mathrm{c}}$ of the gravity of the car body, the potential energy $V_{\mathrm{t}}$ of the gravity of the two bogie frames, and the potential energy $V_{\mathrm{w}}$ of the gravity of the four wheelsets. If zero potential energy for the gravity of the vehicle is assumed when car body, two bogie frames and four wheelsets locate at their static equilibrium position, respectively, the potential-energy relation is

$$
V=U_{\mathrm{v}}+U_{\mathrm{b}}+V_{\mathrm{c}}+V_{\mathrm{t}}+V_{\mathrm{w}}
$$

where

$$
\begin{aligned}
& U_{\mathrm{v}}=\frac{1}{2} k_{\mathrm{v}}\left(y_{\mathrm{t} 1}-y_{\mathrm{c}}+L_{1} \theta_{\mathrm{c}}\right)^{2}+\frac{1}{2} k_{\mathrm{v}}\left(y_{\mathrm{t} 2}-y_{\mathrm{c}}-L_{2} \theta_{\mathrm{c}}\right)^{2} \\
& U_{\mathrm{b}}=\frac{1}{2} \int_{0}^{L} E_{\mathrm{b}} I_{\mathrm{b}}\left(\frac{\partial^{2} y(x, t)}{\partial x^{2}}\right)^{2} d x \\
& V_{\mathrm{c}}=-m_{\mathrm{c}} g\left[\frac{L_{1}}{L_{1}+L_{2}} \cdot \frac{1}{2}\left(y_{\mathrm{w} 3}+y_{\mathrm{w} 4}\right)+\frac{L_{2}}{L_{1}+L_{2}} \cdot \frac{1}{2}\left(y_{\mathrm{w} 1}+y_{\mathrm{w} 2}\right)\right] \\
& V_{\mathrm{t}}=-m_{\mathrm{t}} g \cdot \frac{1}{2}\left(y_{\mathrm{w} 1}+y_{\mathrm{w} 2}\right)-m_{\mathrm{t}} g \cdot \frac{1}{2}\left(y_{\mathrm{w} 3}+y_{\mathrm{w} 4}\right) \\
& V_{\mathrm{w}}=-m_{\mathrm{w}} g \cdot y_{\mathrm{w} 1}-m_{\mathrm{w}} g \cdot y_{\mathrm{w} 2}-m_{\mathrm{w}} g \cdot y_{\mathrm{w} 3}-m_{\mathrm{w}} g \cdot y_{\mathrm{W} 4}
\end{aligned}
$$

Substituting Eqs (20), and (26) into Eq. (38), Eq. (8) into Eq. (39), Eq. (11) into Eqs (40), (41), and (42), then substituting Eqs (38-42) into Eq. (37), one obtains the expression of the total potential energy $V$ for the vehicle-bridge interaction system with DOFs of car body and bridge.

\subsection{The total virtual work performed by nonconservative forces for the vehicle-bridge interaction system}

The total virtual work $\delta W_{\text {nc }}$ performed by nonconservative forces for the vehicle-bridge interaction system

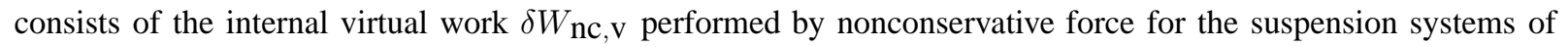
vehicle between bogie frames and car body, and the internal virtual work $\delta W_{\mathrm{nc}, \mathrm{b}}$ performed by nonconservative forces for the beam. It can be written as

$$
\delta W_{\mathrm{nc}}=\delta W_{\mathrm{nc}, \mathrm{v}}+\delta W_{\mathrm{nc}, \mathrm{b}}
$$

For the internal virtual work $\delta W_{\mathrm{nc}, \mathrm{v}}$ performed by nonconservative force for the suspension systems of vehicle between bogie frames and car body, it may be expressed as

$$
\delta W_{\mathrm{nc}, \mathbf{v}}=-c_{\mathrm{v}}\left(\delta y_{\mathrm{t} 1}-\delta y_{\mathrm{c}}+L_{1} \cdot \delta \theta_{\mathrm{c}}\right)\left(\dot{y}_{\mathrm{t} 1}-\dot{y}_{\mathrm{c}}+L_{1} \dot{\theta}_{\mathrm{c}}\right)-c_{\mathrm{v}}\left(\delta y_{\mathrm{t} 2}-\delta y_{\mathrm{c}}-L_{2} \cdot \delta \theta_{\mathrm{c}}\right)\left(\dot{y}_{\mathrm{t} 2}-\dot{y}_{\mathrm{c}}-L_{2} \dot{\theta}_{\mathrm{c}}\right)
$$

For the Bernoulli-Euler beam, it will be assumed that the material of the flexure member obeys the uniaxial stress-strain relation [5]

$$
\sigma(t)=E_{\mathrm{b}}\left[\varepsilon(t)+a_{\mathrm{b}} \dot{\varepsilon}(t)\right]
$$

where $\sigma(t)$ denotes normal stress, $\varepsilon(t)$ denotes normal strain, $\dot{\varepsilon}(t)$ denotes normal strain velocity, and $a_{\mathrm{b}}$ is a damping constant of beam. Using Eq. (45) and the Bernoulli-Euler hypothesis that the normal strains vary linearly over the beam element cross section leads to the moment- curvature relation

$$
M(x, t)=E_{\mathrm{b}} I_{\mathrm{b}}\left[y^{\prime \prime}(x, t)+a_{\mathrm{b}} \dot{y}^{\prime \prime}(x, t)\right]
$$


The first term on the right hand side of Eq. (46) results from the internal conservative forces, which have already been accounted for in the potential-energy term $U_{\mathrm{b}}$, while the second term results from the internal nonconservative forces. The virtual work performed by these nonconservative forces per unit length along the member equals the negative of the product of the nonconservative moment $a_{\mathrm{b}} E_{\mathrm{b}} I_{\mathrm{b}} \dot{y}^{\prime \prime}(x, t)$ times the variation in the curvature $\delta y^{\prime \prime}(x, t)$. Therefore, the virtual work $\delta W_{\mathrm{nc}, \mathrm{b}}$ performed by these internal nonconservative forces for the Bernoulli-Euler beam is

$$
\delta W_{\mathrm{nc}, \mathrm{b}}=-a_{\mathrm{b}} \int_{0}^{L} E_{\mathrm{b}} I_{\mathrm{b}} \dot{y}^{\prime \prime}(x, t) \cdot \delta y^{\prime \prime}(x, t) d x
$$

Substituting $\dot{y}^{\prime \prime}(x, t)=-\sum_{j=1}^{n} \dot{A}_{j} \cdot\left(\frac{j \pi}{L}\right)^{2} \sin (j \pi x / L)$ and $\delta y^{\prime \prime}(x, t)=-\sum_{j=1}^{n} \delta A_{j} \cdot\left(\frac{j \pi}{L}\right)^{2} \sin (j \pi x / L)$ into Eq. (47) gives

$$
\delta W_{\mathrm{nc}, \mathrm{b}}=-\sum_{j=1}^{n}\left[\delta A_{j} \cdot\left(a_{\mathrm{b}} \int_{0}^{L} E_{\mathrm{b}} I_{\mathrm{b}}\left(\frac{j \pi}{L}\right)^{4}\left(\sin \frac{j \pi x}{L}\right)^{2} d x\right) \cdot \dot{A}_{j}\right]
$$

Substituting Eqs (21), and (27) into Eq. (44), then substituting Eqs (44) and (48) into Eq. (43), one obtains the expression of the total virtual work $\delta W_{\mathrm{nc}}$ performed by nonconservative forces for the vehicle-bridge interaction system with DOFs of car body and bridge.

\subsection{The equations of vertical motion of vehicle-bridge interaction system}

Substituting Eqs (32), (37), and (43) with DOFs of car body and bridge into Eq. (1) and rearranging, one obtains the global equations of vertical motion for the entire vehicle-bridge interaction system. The equations can be written in matrix form as

$$
\mathbf{M} \ddot{\mathbf{q}}+\mathbf{C} \dot{\mathbf{q}}+\mathbf{K q}=\mathbf{F}
$$

where $\mathbf{M}, \mathbf{C}$, and $\mathbf{K}$ are the mass, damping, and stiffness matrices for the system, and $\ddot{\mathbf{q}}, \dot{\mathbf{q}}, \mathbf{q}$, and $\mathbf{F}$ are the acceleration, velocity, displacement, and load vectors, respectively. Equation (49) can also be written in sub-matrices form as

$$
\left[\begin{array}{ll}
\mathbf{M}_{\mathbf{V}} & 0 \\
\mathbf{0} & \mathbf{M}^{*}
\end{array}\right]\left\{\begin{array}{l}
\ddot{\mathrm{q}}_{\mathrm{v}} \\
\ddot{\mathrm{A}}
\end{array}\right\}+\left[\begin{array}{ll}
\mathbf{C}_{\mathrm{V}} & \mathbf{C}_{12} \\
\mathbf{C}_{21} & \mathbf{C}^{*}
\end{array}\right]\left\{\begin{array}{l}
\dot{\mathrm{q}}_{\mathrm{v}} \\
\dot{\mathrm{A}}
\end{array}\right\}+\left[\begin{array}{ll}
\mathbf{K}_{\mathrm{V}} & \mathbf{K}_{12} \\
\mathbf{K}_{21} & \mathbf{K}^{*}
\end{array}\right]\left\{\begin{array}{l}
\mathbf{q}_{\mathrm{v}} \\
\mathbf{A}
\end{array}\right\}=\left\{\begin{array}{l}
\mathbf{F}_{\mathbf{v}} \\
\mathbf{F}^{*}
\end{array}\right\}
$$

where the sub-matrices are given as follows

$$
\begin{aligned}
& \mathbf{M}_{\mathbf{V}}=\operatorname{diag}\left[m_{\mathrm{c}} J_{\mathrm{c}}\right] \\
& \mathbf{M}^{*}=\mathbf{M}^{* 1}+\mathbf{M}^{* 2} \\
& \mathbf{M}^{* 1}=\operatorname{diag}\left[\frac{1}{2} \bar{m}_{\mathrm{b}} L \frac{1}{2} \bar{m}_{\mathrm{b}} L \cdots \frac{1}{2} \bar{m}_{\mathrm{b}} L\right] \\
& \mathbf{M}^{* 2}=\left[\begin{array}{llll}
m_{11} & m_{12} & \cdots & m_{1 \mathrm{n}} \\
m_{21} & m_{22} & \cdots & m_{2 \mathrm{n}} \\
\vdots & \vdots & \ddots \\
m_{\mathrm{n} 1} & m_{\mathrm{n} 2} & \cdots & m_{\mathrm{nn}}
\end{array}\right]
\end{aligned}
$$




$$
\begin{aligned}
& m_{j_{1} j_{2}}=\left[\frac{m_{\mathrm{t}}}{4}+\frac{J_{\mathrm{t}}}{4 L_{\mathrm{t}}^{2}}+m_{\mathrm{w}}\right]\left(\sin \frac{j_{1} \pi x_{1}}{L} \sin \frac{j_{2} \pi x_{1}}{L}+\sin \frac{j_{1} \pi x_{2}}{L} \sin \frac{j_{2} \pi x_{2}}{L}+\sin \frac{j_{1} \pi x_{3}}{L} \sin \frac{j_{2} \pi x_{3}}{L}\right. \\
& \left.+\sin \frac{j_{1} \pi x_{4}}{L} \sin \frac{j_{2} \pi x_{4}}{L}\right)+\left[\frac{m_{\mathrm{t}}}{4}-\frac{J_{\mathrm{t}}}{4 L_{\mathrm{t}}^{2}}\right]\left(\sin \frac{j_{1} \pi x_{1}}{L} \sin \frac{j_{2} \pi x_{2}}{L}+\sin \frac{j_{1} \pi x_{2}}{L} \sin \frac{j_{2} \pi x_{1}}{L}\right. \\
& \left.+\sin \frac{j_{1} \pi x_{3}}{L} \sin \frac{j_{2} \pi x_{4}}{L}+\sin \frac{j_{1} \pi x_{4}}{L} \sin \frac{j_{2} \pi x_{3}}{L}\right) \\
& j_{1}, \quad j_{2}=1, \quad 2, \quad \ldots, n \\
& \mathbf{C}_{\mathbf{v}}=\left[\begin{array}{ll}
2 c_{\mathrm{v}} & -c_{\mathrm{v}} L_{1}+c_{\mathrm{v}} L_{2} \\
-c_{\mathrm{v}} L_{1}+c_{\mathrm{v}} L_{2} & c_{\mathrm{v}} L_{1}^{2}+c_{\mathrm{v}} L_{2}^{2}
\end{array}\right] \\
& \mathbf{C}^{*}=\mathbf{C}^{* 1}+\mathbf{C}^{* 2} \\
& \mathbf{C}^{* 1}=\operatorname{diag}\left[a_{\mathrm{b}} \frac{L}{2} E_{b} I_{b}\left(\frac{\pi}{L}\right)^{4} a_{b} \frac{L}{2} E_{b} I_{b}\left(\frac{2 \pi}{L}\right)^{4} \cdots a_{b} \frac{L}{2} E_{b} I_{b}\left(\frac{n \pi}{L}\right)^{4}\right] \\
& \mathbf{C}^{* 2}=\left[\begin{array}{llll}
c_{11} & c_{12} & \cdots & c_{1 \mathrm{n}} \\
c_{21} & c_{22} & \cdots & c_{2 \mathrm{n}} \\
\vdots & \vdots & \ddots & \vdots \\
c_{\mathrm{n} 1} & c_{\mathrm{n} 2} & \cdots & c_{\mathrm{nn}}
\end{array}\right] \\
& c_{j_{1} j_{2}}=\frac{c_{\mathrm{v}}}{4}\left(\sin \frac{j_{1} \pi x_{1}}{L} \sin \frac{j_{2} \pi x_{1}}{L}+\sin \frac{j_{1} \pi x_{2}}{L} \sin \frac{j_{2} \pi x_{2}}{L}+\sin \frac{j_{1} \pi x_{3}}{L} \sin \frac{j_{2} \pi x_{3}}{L}+\sin \frac{j_{1} \pi x_{4}}{L} \sin \frac{j_{2} \pi x_{4}}{L}\right) \\
& +\frac{c_{\mathrm{v}}}{4}\left(\sin \frac{j_{1} \pi x_{1}}{L} \sin \frac{j_{2} \pi x_{2}}{L}+\sin \frac{j_{1} \pi x_{2}}{L} \sin \frac{j_{2} \pi x_{1}}{L}+\sin \frac{j_{1} \pi x_{3}}{L} \sin \frac{j_{2} \pi x_{4}}{L}+\sin \frac{j_{1} \pi x_{4}}{L} \sin \frac{j_{2} \pi x_{3}}{L}\right) \\
& +v \frac{j_{2} \pi}{L}\left[\frac{m_{\mathrm{t}}}{2}+\frac{J_{\mathrm{t}}}{2 L_{\mathrm{t}}^{2}}+2 m_{\mathrm{w}}\right]\left(\sin \frac{j_{1} \pi x_{1}}{L} \cos \frac{j_{2} \pi x_{1}}{L}+\sin \frac{j_{1} \pi x_{2}}{L} \cos \frac{j_{2} \pi x_{2}}{L}+\sin \frac{j_{1} \pi x_{3}}{L} \cos \frac{j_{2} \pi x_{3}}{L}\right. \\
& \left.+\sin \frac{j_{1} \pi x_{4}}{L} \cos \frac{j_{2} \pi x_{4}}{L}\right)+v \frac{j_{2} \pi}{L}\left[\frac{m_{\mathrm{t}}}{2}-\frac{J_{\mathrm{t}}}{2 L_{\mathrm{t}}^{2}}\right]\left(\sin \frac{j_{1} \pi x_{1}}{L} \cos \frac{j_{2} \pi x_{2}}{L}+\sin \frac{j_{1} \pi x_{2}}{L} \cos \frac{j_{2} \pi x_{1}}{L}\right. \\
& \left.+\sin \frac{j_{1} \pi x_{3}}{L} \cos \frac{j_{2} \pi x_{4}}{L}+\sin \frac{j_{1} \pi x_{4}}{L} \cos \frac{j_{2} \pi x_{3}}{L}\right) \\
& j_{1}, \quad j_{2}=1, \quad 2, \quad \ldots, n \\
& \mathbf{C}_{12}=\left[\begin{array}{llll}
\bar{c}_{11} & \bar{c}_{12} & \cdots & \bar{c}_{1 \mathrm{n}} \\
\bar{c}_{21} & \bar{c}_{22} & \cdots & \bar{c}_{2 \mathrm{n}}
\end{array}\right] \\
& \bar{c}_{1 j}=-\frac{c_{\mathrm{v}}}{2}\left(\sin \frac{j \pi x_{1}}{L}+\sin \frac{j \pi x_{2}}{L}+\sin \frac{j \pi x_{3}}{L}+\sin \frac{j \pi x_{4}}{L}\right) \quad j=1, \quad 2, \ldots, n \\
& \bar{c}_{2 j}=\frac{c_{\mathrm{v}}}{2}\left(L_{1} \sin \frac{j \pi x_{1}}{L}+L_{1} \sin \frac{j \pi x_{2}}{L}-L_{2} \sin \frac{j \pi x_{3}}{L}-L_{2} \sin \frac{j \pi x_{4}}{L}\right) \quad j=1, \quad 2, \quad \ldots, n \\
& \mathbf{C}_{21}=\mathbf{C}_{12}^{\mathrm{T}} \\
& \mathbf{K}_{\mathbf{V}}=\left[\begin{array}{lr}
2 k_{\mathrm{v}} & -k_{\mathrm{v}} L_{1}+k_{\mathrm{v}} L_{2} \\
-k_{\mathrm{v}} L_{1}+k_{\mathrm{v}} L_{2} & k_{\mathrm{v}} L_{1}^{2}+k_{\mathrm{v}} L_{2}^{2}
\end{array}\right]
\end{aligned}
$$




$$
\begin{aligned}
& \mathbf{K}^{*}=\mathbf{K}^{* 1}+\mathbf{K}^{* 2} \\
& \mathbf{K}^{* 1}=\operatorname{diag}\left[\frac{L}{2} E_{\mathrm{b}} I_{\mathrm{b}}\left(\frac{\pi}{L}\right)^{4} \frac{L}{2} E_{\mathrm{b}} I_{\mathrm{b}}\left(\frac{2 \pi}{L}\right)^{4} \cdots \frac{L}{2} E_{\mathrm{b}} I_{\mathrm{b}}\left(\frac{n \pi}{L}\right)^{4}\right] \\
& \mathbf{K}^{* 2}=\left[\begin{array}{llll}
k_{11} & k_{12} & \cdots & k_{\mathrm{nn}} \\
k_{21} & k_{22} & \cdots & k_{2 \mathrm{n}} \\
\vdots & \vdots & \ddots & \vdots \\
k_{\mathrm{n} 1} & k_{\mathrm{n} 2} & \cdots & k_{\mathrm{nn}}
\end{array}\right] \\
& k_{j_{1} j_{2}}=\left[\frac{k_{\mathrm{v}}}{4}-\frac{m_{\mathrm{t}}}{4} v^{2}\left(\frac{j_{2} \pi}{L}\right)^{2}-\frac{J_{\mathrm{t}}}{4 L_{\mathrm{t}}^{2}} v^{2}\left(\frac{j_{2} \pi}{L}\right)^{2}-m_{\mathrm{w}} v^{2}\left(\frac{j_{2} \pi}{L}\right)^{2}\right]\left(\sin \frac{j_{1} \pi x_{1}}{L} \sin \frac{j_{2} \pi x_{1}}{L}\right. \\
& \left.+\sin \frac{j_{1} \pi x_{2}}{L} \sin \frac{j_{2} \pi x_{2}}{L}+\sin \frac{j_{1} \pi x_{3}}{L} \sin \frac{j_{2} \pi x_{3}}{L}+\sin \frac{j_{1} \pi x_{4}}{L} \sin \frac{j_{2} \pi x_{4}}{L}\right)+ \\
& {\left[\frac{k_{\mathrm{v}}}{4}-\frac{m_{\mathrm{t}}}{4} v^{2}\left(\frac{j_{2} \pi}{L}\right)^{2}+\frac{J_{\mathrm{t}}}{4 L_{\mathrm{t}}^{2}} v^{2}\left(\frac{j_{2} \pi}{L}\right)^{2}\right]\left(\sin \frac{j_{1} \pi x_{1}}{L} \sin \frac{j_{2} \pi x_{2}}{L}+\sin \frac{j_{1} \pi x_{2}}{L} \sin \frac{j_{2} \pi x_{1}}{L}\right.} \\
& \left.+\sin \frac{j_{1} \pi x_{3}}{L} \sin \frac{j_{2} \pi x_{4}}{L}+\sin \frac{j_{1} \pi x_{4}}{L} \sin \frac{j_{2} \pi x_{3}}{L}\right)+ \\
& \frac{j_{2} \pi}{L}\left(\frac{c_{\mathrm{v}} v}{4}+\frac{m_{\mathrm{t}} a}{4}+\frac{J_{\mathrm{t}} a}{4 L_{\mathrm{t}}^{2}}+m_{\mathrm{w}} a\right)\left(\sin \frac{j_{1} \pi x_{1}}{L} \cos \frac{j_{2} \pi x_{1}}{L}+\sin \frac{j_{1} \pi x_{2}}{L} \cos \frac{j_{2} \pi x_{2}}{L}\right. \\
& \left.+\sin \frac{j_{1} \pi x_{3}}{L} \cos \frac{j_{2} \pi x_{3}}{L}+\sin \frac{j_{1} \pi x_{4}}{L} \cos \frac{j_{2} \pi x_{4}}{L}\right)+ \\
& \frac{j_{2} \pi}{L}\left(\frac{c_{\mathrm{v}} v}{4}+\frac{m_{\mathrm{t}} a}{4}-\frac{J_{\mathrm{t}} a}{4 L_{\mathrm{t}}^{2}}\right)\left(\sin \frac{j_{1} \pi x_{1}}{L} \cos \frac{j_{2} \pi x_{2}}{L}+\sin \frac{j_{1} \pi x_{2}}{L} \cos \frac{j_{2} \pi x_{1}}{L}\right. \\
& \left.+\sin \frac{j_{1} \pi x_{3}}{L} \cos \frac{j_{2} \pi x_{4}}{L}+\sin \frac{j_{1} \pi x_{4}}{L} \cos \frac{j_{2} \pi x_{3}}{L}\right) \\
& j_{1}, \quad j_{2}=1, \quad 2, \quad \ldots, n \\
& \mathbf{K}_{12}=\left[\begin{array}{llll}
\bar{k}_{11} & \bar{k}_{12} & \cdots & \bar{k}_{1 \mathrm{n}} \\
\bar{k}_{21} & \bar{k}_{22} & \cdots & \bar{k}_{2 \mathrm{n}}
\end{array}\right] \\
& \bar{k}_{1 j}=-\frac{k_{\mathrm{v}}}{2}\left(\sin \frac{j \pi x_{1}}{L}+\sin \frac{j \pi x_{2}}{L}+\sin \frac{j \pi x_{3}}{L}+\sin \frac{j \pi x_{4}}{L}\right) \\
& -\frac{j c_{\mathrm{v}} v \pi}{2 L}\left(\cos \frac{j \pi x_{1}}{L}+\cos \frac{j \pi x_{2}}{L}+\cos \frac{j \pi x_{3}}{L}+\cos \frac{j \pi x_{4}}{L}\right) \quad j=1, \quad 2, \ldots, n \\
& \bar{k}_{2 j}=\frac{k_{\mathrm{v}}}{2}\left(L_{1} \sin \frac{j \pi x_{1}}{L}+L_{1} \sin \frac{j \pi x_{2}}{L}-L_{2} \sin \frac{j \pi x_{3}}{L}-L_{2} \sin \frac{j \pi x_{4}}{L}\right)+ \\
& \frac{j c_{\mathrm{v}} v \pi}{2 L}\left(L_{1} \cos \frac{j \pi x_{1}}{L}+L_{1} \cos \frac{j \pi x_{2}}{L}-L_{2} \cos \frac{j \pi x_{3}}{L}-L_{2} \cos \frac{j \pi x_{4}}{L}\right) \\
& j=1,2, \ldots, n
\end{aligned}
$$




$$
\begin{aligned}
& \mathbf{K}_{21}=\left[\begin{array}{ll}
\hat{k}_{11} & \hat{k}_{12} \\
\hat{k}_{21} & \hat{k}_{22} \\
\vdots & \vdots \\
\hat{k}_{\mathrm{n} 1} & \hat{k}_{\mathrm{n} 2}
\end{array}\right] \\
& \hat{k}_{j 1}=-\frac{k_{\mathrm{v}}}{2}\left(\sin \frac{j \pi x_{1}}{L}+\sin \frac{j \pi x_{2}}{L}+\sin \frac{j \pi x_{3}}{L}+\sin \frac{j \pi x_{4}}{L}\right) \quad j=1,2, \ldots, n \\
& \hat{k}_{j 2}=\frac{k_{\mathrm{v}}}{2}\left(L_{1} \sin \frac{j \pi x_{1}}{L}+L_{1} \sin \frac{j \pi x_{2}}{L}-L_{2} \sin \frac{j \pi x_{3}}{L}-L_{2} \sin \frac{j \pi x_{4}}{L}\right) \quad j=1, \quad 2, \quad \ldots, n \\
& \mathbf{q v}=\left[y_{\mathrm{c}} \theta_{\mathrm{c}}\right]^{\mathrm{T}} \\
& \mathbf{A}=\left[A_{1} A_{2} \cdots A_{\mathrm{n}}\right]^{\mathrm{T}} \\
& \mathbf{F}_{\mathbf{v}}=\left\{\begin{array}{l}
F_{\mathbf{V} 1}(t) \\
F_{\mathrm{V} 2}(t)
\end{array}\right\} \\
& F_{\mathrm{V} 1}(t)=\frac{k_{\mathrm{v}}}{2}\left[r_{\left(x_{1}\right)}+r_{\left(x_{2}\right)}+r_{\left(x_{3}\right)}+r_{\left(x_{4}\right)}\right]+\frac{c_{\mathrm{v}} v}{2}\left[r_{\left(x_{1}\right)}^{\prime}+r_{\left(x_{2}\right)}^{\prime}+r_{\left(x_{3}\right)}^{\prime}+r_{\left(x_{4}\right)}^{\prime}\right] \\
& F_{\mathrm{V} 2}(t)=-\frac{k_{\mathrm{v}}}{2}\left[L_{1} r_{\left(x_{1}\right)}+L_{1} r_{\left(x_{2}\right)}-L_{2} r_{\left(x_{3}\right)}-L_{2} r_{\left(x_{4}\right)}\right]-\frac{c_{\mathrm{v}} v}{2}\left[L_{1} r_{\left(x_{1}\right)}^{\prime}+L_{1} r_{\left(x_{2}\right)}^{\prime}-L_{2} r_{\left(x_{3}\right)}^{\prime}-L_{2} r_{\left(x_{4}\right)}^{\prime}\right] \\
& \mathbf{F}^{*}=\left[F_{1}(t) F_{2}(t) \cdots F_{\mathrm{n}}(t)\right]^{\mathrm{T}} \\
& F_{j}(t)=\left(m_{\mathrm{w}} g+\frac{1}{2} m_{\mathrm{t}} g+\frac{1}{2} m_{\mathrm{c}} g \frac{L_{2}}{L_{1}+L_{2}}\right)\left(\sin \frac{j \pi x_{1}}{L}+\sin \frac{j \pi x_{2}}{L}\right)+ \\
& \left(m_{\mathrm{w}} g+\frac{1}{2} m_{\mathrm{t}} g+\frac{1}{2} m_{\mathrm{c}} g \frac{L_{1}}{L_{1}+L_{2}}\right)\left(\sin \frac{j \pi x_{3}}{L}+\sin \frac{j \pi x_{4}}{L}\right) \\
& -\frac{1}{4}\left[k_{\mathrm{v}} r_{\left(x_{1}\right)}+k_{\mathrm{v}} r_{\left(x_{2}\right)}+c_{\mathrm{v}} v r_{\left(x_{1}\right)}^{\prime}+c_{\mathrm{v}} v r_{\left(x_{2}\right)}^{\prime}+m_{\mathrm{t}}\left(v^{2} \cdot r_{\left(x_{1}\right)}^{\prime \prime}+a \cdot r_{\left(x_{1}\right)}^{\prime}+v^{2} \cdot r_{\left(x_{2}\right)}^{\prime \prime}+a \cdot r_{\left(x_{2}\right)}^{\prime}\right)\right. \\
& \left.+\frac{J_{\mathrm{t}}}{L_{\mathrm{t}}^{2}}\left(v^{2} \cdot r_{\left(x_{1}\right)}^{\prime \prime}+a \cdot r_{\left(x_{1}\right)}^{\prime}-v^{2} \cdot r_{\left(x_{2}\right)}^{\prime \prime}-a \cdot r_{\left(x_{2}\right)}^{\prime}\right)+4 m_{\mathrm{w}}\left(v^{2} \cdot r_{\left(x_{1}\right)}^{\prime \prime}+a \cdot r_{\left(x_{1}\right)}^{\prime}\right)\right] \sin \frac{j \pi x_{1}}{L} \\
& -\frac{1}{4}\left[k_{\mathrm{v}} r_{\left(x_{1}\right)}+k_{\mathrm{v}} r_{\left(x_{2}\right)}+c_{\mathrm{v}} v r_{\left(x_{1}\right)}^{\prime}+c_{\mathrm{v}} v r_{\left(x_{2}\right)}^{\prime}+m_{\mathrm{t}}\left(v^{2} \cdot r_{\left(x_{1}\right)}^{\prime \prime}+a \cdot r_{\left(x_{1}\right)}^{\prime}+v^{2} \cdot r_{\left(x_{2}\right)}^{\prime \prime}+a \cdot r_{\left(x_{2}\right)}^{\prime}\right)\right. \\
& \left.-\frac{J_{\mathrm{t}}}{L_{\mathrm{t}}^{2}}\left(v^{2} \cdot r_{\left(x_{1}\right)}^{\prime \prime}+a \cdot r_{\left(x_{1}\right)}^{\prime}-v^{2} \cdot r_{\left(x_{2}\right)}^{\prime \prime}-a \cdot r_{\left(x_{2}\right)}^{\prime}\right)+4 m_{\mathrm{w}}\left(v^{2} \cdot r_{\left(x_{2}\right)}^{\prime \prime}+a \cdot r_{\left(x_{2}\right)}^{\prime}\right)\right] \sin \frac{j \pi x_{2}}{L} \\
& -\frac{1}{4}\left[k_{\mathrm{v}} r_{\left(x_{3}\right)}+k_{\mathrm{v}} r_{\left(x_{4}\right)}+c_{\mathrm{v}} v r_{\left(x_{3}\right)}^{\prime}+c_{\mathrm{v}} v r_{\left(x_{4}\right)}^{\prime}+m_{\mathrm{t}}\left(v^{2} \cdot r_{\left(x_{3}\right)}^{\prime \prime}+a \cdot r_{\left(x_{3}\right)}^{\prime}+v^{2} \cdot r_{\left(x_{4}\right)}^{\prime \prime}+a \cdot r_{\left(x_{4}\right)}^{\prime}\right)\right. \\
& \left.+\frac{J_{\mathrm{t}}}{L_{\mathrm{t}}^{2}}\left(v^{2} \cdot r_{\left(x_{3}\right)}^{\prime \prime}+a \cdot r_{\left(x_{3}\right)}^{\prime}-v^{2} \cdot r_{\left(x_{4}\right)}^{\prime \prime}-a \cdot r_{\left(x_{4}\right)}^{\prime}\right)+4 m_{\mathrm{w}}\left(v^{2} \cdot r_{\left(x_{3}\right)}^{\prime \prime}+a \cdot r_{\left(x_{3}\right)}^{\prime}\right)\right] \sin \frac{j \pi x_{3}}{L} \\
& -\frac{1}{4}\left[k_{\mathrm{v}} r_{\left(x_{3}\right)}+k_{\mathrm{v}} r_{\left(x_{4}\right)}+c_{\mathrm{v}} v r_{\left(x_{3}\right)}^{\prime}+c_{\mathrm{v}} v r_{\left(x_{4}\right)}^{\prime}+m_{\mathrm{t}}\left(v^{2} \cdot r_{\left(x_{3}\right)}^{\prime \prime}+a \cdot r_{\left(x_{3}\right)}^{\prime}+v^{2} \cdot r_{\left(x_{4}\right)}^{\prime \prime}+a \cdot r_{\left(x_{4}\right)}^{\prime}\right)\right. \\
& \left.-\frac{J_{\mathrm{t}}}{L_{\mathrm{t}}^{2}}\left(v^{2} \cdot r_{\left(x_{3}\right)}^{\prime \prime}+a \cdot r_{\left(x_{3}\right)}^{\prime}-v^{2} \cdot r_{\left(x_{4}\right)}^{\prime \prime}-a \cdot r_{\left(x_{4}\right)}^{\prime}\right)+4 m_{\mathrm{w}}\left(v^{2} \cdot r_{\left(x_{4}\right)}^{\prime \prime}+a \cdot r_{\left(x_{4}\right)}^{\prime}\right)\right] \sin \frac{j \pi x_{4}}{L} \\
& j=1, \quad 2, \ldots, n
\end{aligned}
$$




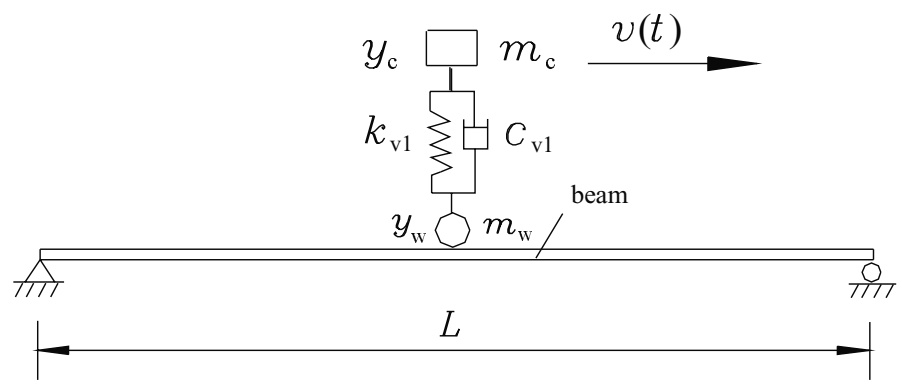

Fig. 2. A simply supported beam subjected to a moving one-axle vehicle.

in which, superscript $\mathrm{T}$ represents the transpose.

It should be noted that the matrices of $\mathbf{C}^{*}$ and $\mathbf{K}^{*}$ in Eq. (50) are unsymmetrical, the matrices of $\mathbf{M}^{*}, \mathbf{C}_{12}, \mathbf{C}_{21}$, $\mathbf{C}^{*}, \mathbf{K}_{12}, \mathbf{K}_{21}$, and $\mathbf{K}^{*}$, and the vectors of $\mathbf{F}_{c}$ and $\mathbf{F}^{*}$ are time-dependent; the matrices of $\mathbf{C}$ and $\mathbf{K}$ in Eq. (49) are unsymmetrical, and the matrices of $\mathbf{M}, \mathbf{C}$, and $\mathbf{K}$, and the vectors of $\mathbf{F}$ are time-dependent.

Equations (49) or (50) can be solved by direct time integration method such as Newmark $\beta$ method or Wilson- $\theta$ method, to obtain simultaneously the dynamic responses of vehicle, of contact forces, and of bridge. This equation has been written on the assumption that four wheelsets of vehicle are acting on the bridge. If a certain wheelsets of vehicle are acting on the bridge, Eq. (50) should be modified. For example, if only the front wheelset of the front bogie of vehicle is on the bridge, the corresponding terms including $x_{1}, x_{2}$ and $x_{3}$ in Eq. (50) should be deleted.

Conventionally, the structural damping has been computed on the structure level. Based on the definition of Rayleigh damping, the damping term $\mathbf{C}^{* 1}$ of the bridge in Eq. (50) is computed as follows:

$$
\mathbf{C}^{* 1}=\alpha \cdot \mathbf{M}^{* 1}+\beta \cdot \mathbf{K}^{* 1}
$$

Given the damping ratio $\zeta$, the two coefficients $\alpha$ and $\beta$ can be determined as $\alpha=2 \zeta \omega_{1} \omega_{2} /\left(\omega_{1}+\omega_{2}\right), \beta=$ $2 \zeta /\left(\omega_{1}+\omega_{2}\right)$, where $\omega_{1}$ and $\omega_{2}$ are the first two circular frequencies of vibration of the bridge.

\section{Verification of the proposed procedure}

In this section, the equations of motion derived for the vehicle-bridge interaction system and the associated computer program will be verified through the study of an example.

Consider a simply supported Bernoulli-Euler beam of length $L$ subjected to a moving one-axle vehicle with constant velocity (Fig. 2). The following data are assumed for the vehicle: $m_{\mathrm{c}}=5750 \mathrm{~kg}, k_{\mathrm{V} 1}=1.595 \times 10^{6} \mathrm{~N} / \mathrm{m}$, $c_{\mathrm{V} 1}=0 \mathrm{~N} \cdot \mathrm{s} / \mathrm{m}, m_{\mathrm{w}}=0 \mathrm{~kg}, v=27.78 \mathrm{~m} / \mathrm{s}$, and $a=0 \mathrm{~m} / \mathrm{s}^{2}$. The following data are assumed for the beam: $E_{\mathrm{b}}$ (Young's modulus) $=2.87 \times 10^{9} \mathrm{~Pa}, I_{\mathrm{b}}$ (moment of inertia) $=2.90 \mathrm{~m}^{4}, \bar{m}_{\mathrm{b}}$ (mass per unit length) $=2.303$ $\times 10^{3} \mathrm{~kg} / \mathrm{m}, L$ (span length) $=30 \mathrm{~m}$, with the damping of the beam being neglected and the smoothness of the beam surface being assumed. The first computed circular frequency for beam is $\omega_{1}=20.847 \mathrm{rad} / \mathrm{s}$. To make use of the proposed procedure, the following data are assumed for the vehicle and beam: $m_{\mathrm{c}}=5750 \mathrm{~kg}, J_{\mathrm{c}}=$ $0.1 \mathrm{~kg} \cdot \mathrm{m}^{2}, m_{\mathrm{t}}=0.01 \mathrm{~kg}, J_{\mathrm{t}}=0.1 \mathrm{~kg} \cdot \mathrm{m}^{2}, m_{\mathrm{w}}=0.01 \mathrm{~kg}, k_{\mathrm{v}}=0.5 k_{\mathrm{V} 1}=7.975 \times 10^{5} \mathrm{~N} / \mathrm{m}, c_{\mathrm{v}}=0 \mathrm{~N} \cdot \mathrm{s} / \mathrm{m}$, and $L_{1}=L_{2}=L_{\mathrm{t}}=0 \mathrm{~m} ; E_{\mathrm{b}}=2.87 \times 10^{9} \mathrm{~Pa}, I_{\mathrm{b}}=2.90 \mathrm{~m}^{4}, \bar{m}_{\mathrm{b}}=2.303 \times 10^{3} \mathrm{~kg} / \mathrm{m}$, and $\zeta=0$. It should be noted that $L_{1} /\left(L_{1}+L_{2}\right)$ and $L_{2} /\left(L_{1}+L_{2}\right)$ abovementioned in Eq. (50) are to be replaced by 0.5 and the terms whose denominators contain $L_{\mathrm{t}}^{2}$ are to be replaced by zero. In the modal analysis method, the first mode for the beam is used. The equations of motion for the vehicle-bridge interaction system are solved by the Wilson- $\theta$ method, with $\theta=1.4$ and time step $\Delta t=0.005 \mathrm{~s}$. It is assumed that the one-axle vehicle enters the beam at $t=0 \mathrm{~s}$, the vehicle will leave the beam at $t=1.08 \mathrm{~s}$ for the velocity $27.78 \mathrm{~m} / \mathrm{s}$. In addition, the vehicle and the beam are at rest at $t=0 \mathrm{~s}$. The time-history responses of sprung mass and of the midpoint of beam have been plotted in Figs 3-6.

From Figs 3-6, one observes that the vertical displacement of the sprung mass, the vertical acceleration of the sprung mass, the midpoint vertical displacement of the bridge, and the midpoint vertical acceleration computed by the present method agrees well respectively with those computed by the modal analysis method (MAM) [11] considering the first mode for analyzing dynamic responses of a single-span simply supported bridge under a moving one-axle vehicle. This example serves to illustrate the reliability of the proposed procedure. 


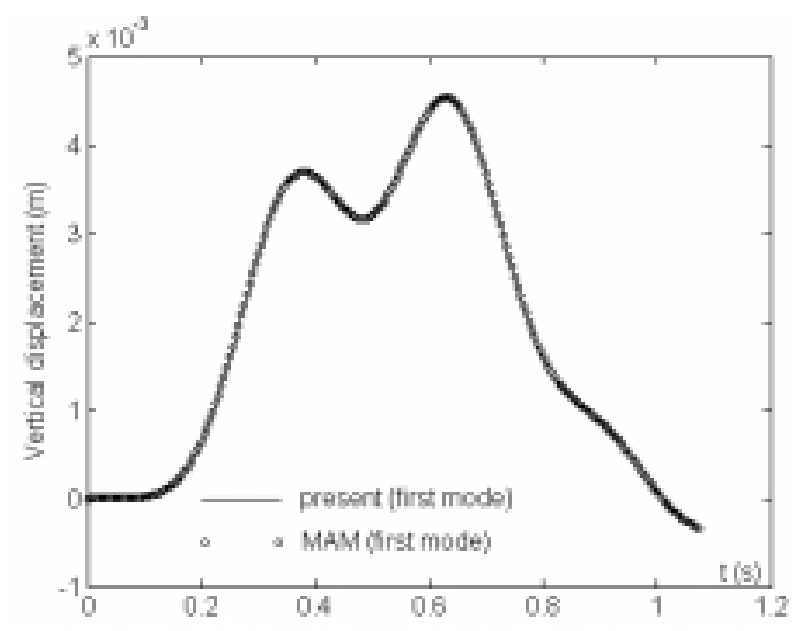

Fig. 3. The time history of vertical displacement of sprung mass.

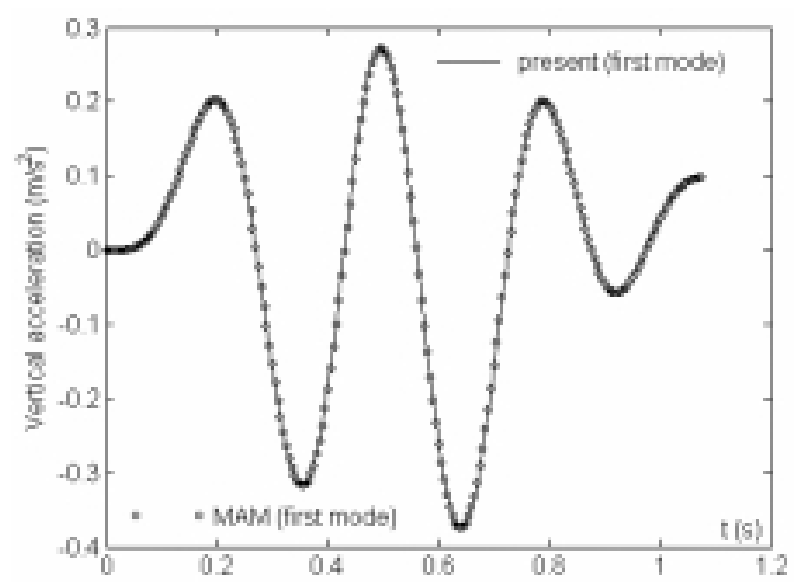

Fig. 4. The time history of vertical acceleration of sprung mass.

\section{Application of the proposed procedure}

In this section, two illustrated numerical examples are investigated to analyze the effect of different beam mode numbers and various surface irregularities of beam on the dynamic responses of the vehicle-bridge interaction system. In the following examples, the Wilson- $\theta$ method and time step $\Delta t=0.005 \mathrm{~s}$ are used to solve the equations of motion for this interaction system.

\subsection{The effect of beam mode number}

In this example, the effect of beam mode numbers including one, three, five and fifteen on the dynamic responses of the vehicle-bridge interaction system is studied. The following data are adopted for the vehicle: $m_{\mathrm{c}}=7.7 \times$ $10^{4} \mathrm{~kg}, J_{\mathrm{c}}=1.2 \times 10^{6} \mathrm{~kg} \cdot \mathrm{m}^{2}, k_{\mathrm{v}}=1.064 \times 10^{7} \mathrm{~N} / \mathrm{m}, c_{\mathrm{v}}=1.4 \times 10^{5} \mathrm{~N} \cdot \mathrm{s} / \mathrm{m}, m_{\mathrm{t}}=1.13 \times 10^{3} \mathrm{~kg}, J_{\mathrm{t}}=760 \mathrm{~kg} \cdot \mathrm{m}^{2}$, $m_{\mathrm{w}}=1.2 \times 10^{3} \mathrm{~kg}, L_{1}=4.25 \mathrm{~m}, L_{2}=4.25 \mathrm{~m}, L_{\mathrm{t}}=0.75 \mathrm{~m}$, and vehicle constant velocity $v=27.78 \mathrm{~m} / \mathrm{s}$. The following data are adopted for the simply supported bridge: $L=20 \mathrm{~m}, E_{\mathrm{b}}=2.943 \times 10^{10} \mathrm{~Pa}, I_{\mathrm{b}}=3.81 \mathrm{~m}^{4}, \bar{m}_{\mathrm{b}}=$ $3.4088 \times 10^{4} \mathrm{~kg} / \mathrm{m}$, and the damping ratio of bridge $\zeta=0.025$; and that the surface of beam is smooth is assumed. It is assumed that the front wheelset of front bogie enters the bridge at $t=0 \mathrm{~s}$, the rear wheelset of rear bogie will leave the bridge at $t=1.08 \mathrm{~s}$ for the velocity $27.78 \mathrm{~m} / \mathrm{s}$. In addition, the vehicle and the beam are at rest at $t=0 \mathrm{~s}$. 


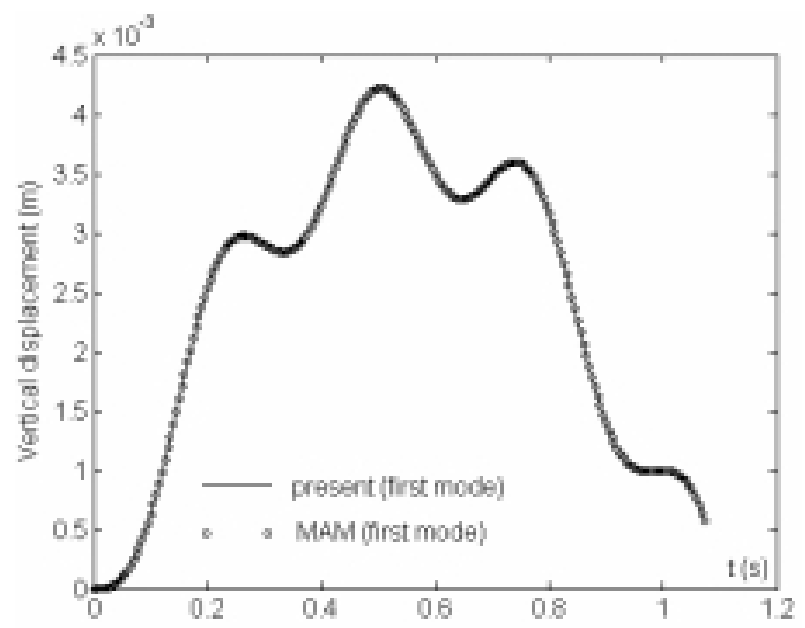

Fig. 5. The time history of vertical displacement of midpoint of beam.

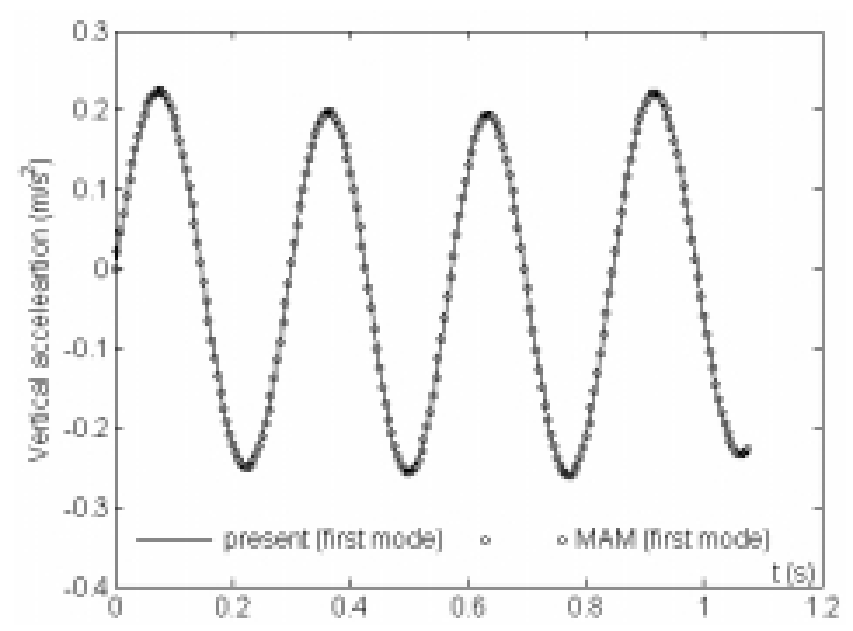

Fig. 6. The time history of vertical acceleration of midpoint of beam.

The dynamic responses of the vehicle-bridge interaction system with various beam mode numbers mentioned above are shown in Table 1. It can be found from Table 1 that the contribution of the first mode in the response considerably exceeds the contribution of the others. The different between the maximum vertical displacement of the midpoint of beam obtained from $n=1$ and that obtained from $n=15$ is about 0.7 percent. However, the difference between the maximum bending moment of the midpoint of beam obtained from $n=1$ and that obtained from $n=15$ is about 8.9 percent. This is due to the beam deflection converges very fast and the computed bending moment is proportional to the second derivative of the deflection with respect to coordinate $x$.

\subsection{The effect of surface irregularity of beam}

To evaluate the effect of surface irregularity of beam on the dynamic responses of the vehicle-bridge interaction system, the dynamic responses of this system for a simply supported bridge with various isolated surface irregularities of beam are investigated. As shown in Fig. 7, the irregularity function proposed in reference [6] for the vertical profile of the bridge is adopted:

$$
r(x)=\frac{1}{2} \bar{a}\left(1-\cos 2 \pi x / l_{a}\right)
$$


Table 1

The dynamic responses of the vehicle-bridge interaction system with various beam mode numbers

\begin{tabular}{lllll}
\hline mode & $n=1$ & $n=3$ & $n=5$ & $n=15$ \\
\hline $\max y_{\text {car }}\left(\times 10^{-4} \mathrm{~m}\right)$ & 8.994 & 9.045 & 9.074 & 9.075 \\
$\max a_{\mathrm{car}}\left(\times 10^{-2} \mathrm{~m} / \mathrm{s}^{2}\right)$ & 7.264 & 7.607 & 7.617 & 7.612 \\
$\min F_{\mathrm{rr}}\left(\times 10^{5} \mathrm{~N}\right)$ & 2.045 & 2.046 & 2.046 & 2.046 \\
$\max F_{\mathrm{rr}}\left(\times 10^{5} \mathrm{~N}\right)$ & 2.076 & 2.076 & 2.076 & 2.076 \\
$\max y_{\mathrm{b}}\left(\times 10^{-4} \mathrm{~m}\right)$ & 9.707 & 9.652 & 9.639 & 9.640 \\
$\max a_{\mathrm{b}}\left(\mathrm{m} / \mathrm{s}^{2}\right)$ & 0.1080 & 0.1121 & 0.1119 & 0.1119 \\
$\max M_{\mathrm{b}}\left(\times 10^{6} \mathrm{~N} \cdot \mathrm{m}\right)$ & 2.686 & 2.548 & 2.470 & 2.467
\end{tabular}

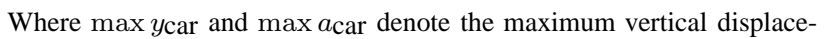
ment and acceleration of the centre of gravity of car body, respectively; $\min F_{\mathrm{rr}}$ and $\max F_{\mathrm{rr}}$ denote the minimum and maximum contact forces between the rear wheelset of rear bogie and beam, respectively; and $\max y_{\mathrm{b}}, \max a_{\mathrm{b}}$ and $\max M_{\mathrm{b}}$ denote the maximum vertical displacement, acceleration and bending moment of the midpoint of beam, respectively.

Table 2

The dynamic responses of the vehicle-bridge interaction system with various irregularity lengths of beam

\begin{tabular}{|c|c|c|c|c|c|c|c|c|c|}
\hline$l_{a}$ & $0.5 \mathrm{~m}$ & $1.0 \mathrm{~m}$ & $1.5 \mathrm{~m}$ & $3.0 \mathrm{~m}$ & $7.0 \mathrm{~m}$ & $8.5 \mathrm{~m}$ & $10.0 \mathrm{~m}$ & $12.0 \mathrm{~m}$ & Smooth surface \\
\hline $\max y \operatorname{car}\left(\times 10^{-4} \mathrm{~m}\right)$ & 9.210 & 8.906 & 8.673 & 8.346 & 13.31 & 14.86 & 15.67 & 15.30 & 8.994 \\
\hline $\max a_{\text {car }}\left(\mathrm{m} / \mathrm{s}^{2}\right)$ & 0.3448 & 0.2119 & 0.1783 & 0.1389 & 0.3274 & 0.3671 & 0.3867 & 0.3887 & 0.07264 \\
\hline $\min F_{\mathrm{rr}}\left(\times 10^{5} \mathrm{~N}\right)$ & 0.3024 & 1.640 & 1.890 & 2.026 & 1.972 & 1.963 & 1.961 & 1.964 & 2.045 \\
\hline $\max F_{\mathrm{rr}}\left(\times 10^{5} \mathrm{~N}\right)$ & 3.796 & 2.479 & 2.250 & 2.122 & 2.136 & 2.136 & 2.132 & 2.130 & 2.076 \\
\hline $\max y_{\mathrm{b}}\left(\times 10^{-4} \mathrm{~m}\right)$ & 9.806 & 9.765 & 9.756 & 9.747 & 9.568 & 9.430 & 9.354 & 9.381 & 9.707 \\
\hline $\max a_{\mathrm{b}}\left(\mathrm{m} / \mathrm{s}^{2}\right)$ & 0.6997 & 0.2212 & 0.1331 & 0.1266 & 0.1135 & 0.110 & 0.1116 & 0.1159 & 0.108 \\
\hline $\max M_{\mathrm{b}}\left(\times 10^{6} \mathrm{~N} \cdot \mathrm{m}\right)$ & 2.713 & 2.702 & 2.699 & 2.697 & 2.647 & 2.609 & 2.588 & 2.595 & 2.686 \\
\hline
\end{tabular}

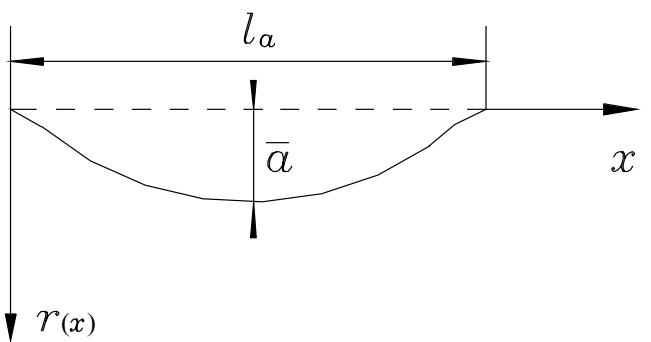

Fig. 7. Model of irregularity on bridge surface.

where, $x$ is the along-bridge distance, $\bar{a}$ is maximum depth of bridge irregularity, and $l_{a}$ is the length of bridge irregularity. For this example, $\bar{a}=1.0 \times 10^{-3} \mathrm{~m}$ and $l_{a}=0.5,1.0,1.5,3.0,7.0,8.5,10.0$ and $12.0 \mathrm{~m}$ are used, respectively, for the isolated bridge irregularity. It is assumed that the point of maximum depth of bridge irregularity locates at the midpoint of the bridge. All the parameters of the vehicle and the bridge are same as those in Section 5.1. In the modal analysis method, the first mode for the beam is used.

Table 2 shows the dynamic responses of the vehicle-bridge interaction system with various irregularity lengths of bridge. In addition, the dynamic responses of the vehicle-bridge interaction system with smooth bridge surface are listed in the last column in Table 2.

As shown in Table 2, the effect of the surface irregularities of bridge on the vertical displacement of bridge midpoint and the bending moment of the cross section of bridge midpoint is insignificant. However, the same is not true for the effect of the surface irregularities of bridge on the vertical displacement and vertical acceleration of the centre of gravity of car body, on the contact force between the rear wheelset of rear bogie and bridge, and on the vertical acceleration of midpoint of bridge. When the value of $l_{a}$ is less than $7.0 \mathrm{~m}$ (the distance of two central wheelsets of vehicle), the effect of the value of $l_{a}$ on the vertical displacement of the centre of gravity of car body is insignificant; with the decrease of the value of $l_{a}$, the vertical acceleration of the centre of gravity of car body, the 
maximum contact force between the rear wheelset of rear bogie and bridge, and the vertical acceleration of midpoint of bridge increase, while the minimum contact force between the rear wheelset of rear bogie and bridge decreases. When the value of $l_{a}$ is more than $7.0 \mathrm{~m}$, the vertical displacement and the vertical acceleration of the centre of gravity of car body almost increase with the increase of the value of $l_{a}$; the effect of the value of $l_{a}$ on the contact force and on the vertical acceleration of midpoint of bridge is insignificant.

\section{Concluding remarks}

Based on energy approach and superimposing modes technique, the equations of motion in matrix form for the interaction system between a railway freight vehicle with 10 DOFs and a simply supported bridge are derived, in which the dynamic contact forces between vehicle and bridge are considered as internal forces. The resulting equations of motion have time-dependent mass, damping, and stiffness matrices, and time-dependent load vector. The damping matrix and stiffness matrix for this system are unsymmetrical. The equations of motion for this system are solved by direct time integration method, to obtain simultaneously the dynamic responses of vehicle, contact forces and bridge. A computer program has been developed for analyzing this system. A numerical example confirms the usefulness of the proposed procedure and two numerical examples illustrate the application of the proposed procedure.

From the numerical results obtained in this work, two conclusions can be reached:

(1). The contribution of the first mode of the bridge in the response considerably exceeds the contribution of the others.

(2). The effect of the surface irregularities of bridge on the vertical displacement of bridge and the bending moment of the cross section of bridge is insignificant. However, the same is not true for the effect of the surface irregularities of bridge on the vertical displacement and vertical acceleration of the centre of gravity of car body, on the contact force between the wheelset and the bridge and on the vertical acceleration of bridge.

\section{Acknowledgement}

The authors would like to thank Dr Zhikun Hou, Worcester Polytechnic Institute and the two unknown reviewers for their valuable and helpful comments.

\section{References}

[1] F.T.K. Au, J.J. Wang and Y.K. Cheung, Impact study of cable-stayed bridge under railway traffic using various models, Journal of Sound and Vibration 240(3) (2001), 447-465.

[2] F.T.K. Au, J.J. Wang and Y.K. Cheung, Impact study of cable-stayed railway bridges with random rail irregularities, Engineering Structures 24(5) (2002), 529-541.

[3] K.J. Bathe and E.L. Wilson, Numerical Methods in Finite Element Analysis, Englewood Cliffs, NJ: Prentice-Hall, 1976.

[4] J.M. Biggs, Introduction to Structural Dynamics, New York: McGraw-Hill, Inc., 1964.

[5] R.W. Clough and J. Penzien, Dynamics of Structures (2nd edition), New York: McGraw-Hill, Inc., 1993.

[6] L. Frýba, Vibration of Solids and Structures under Moving Loads (3rd edition), Prague: Academia, 1999.

[7] J.L. Humar and A.H. Kashif, Dynamic response analysis of slab-type bridges, Journal of Structural Engineering, ASCE 121(1) (1995), 48-62.

[8] Y.H. Lin and M.W. Trethewey, Finite element analysis of elastic beams subjected to moving dynamic loads, Journal of Sound and Vibration 136(2) (1990), 323-342.

[9] P. Lou and Q.Y. Zeng, Finite element based formulations for vehicle-bridge interaction system considering vertical motion, Journal of Structural Engineering 32(4) (2005), 261-273.

[10] N.M. Newmark, A method of computation for structural dynamics, Journal of the Engineering Mechanics Division, Proceedings of the American Society of Civil Engineers 85(3) (1959), 67-94.

[11] M. Olsson, Finite element, modal co-ordinate analysis of structures subjected to moving loads, Journal of Sound and Vibration 99(1) (1985), 1-12.

[12] M. Paz, Modified dynamic condensation method, Journal of Structural Engineering, ASCE 115(1) (1985), 234-238.

[13] S. Timoshenko, D.H. Young and W. Weaver, Jr., Vibration Problems in Engineering (4th edition), New York: John Wiley \& Sons 1974. 
[14] R.K. Wen, Dynamic response of beams traversed by two-axle loads, Journal of the Engineering Mechanics Division, Proceedings of the American Society of Civil Engineers 86(5) (1960), 91-111.

[15] Y.B. Yang, C.H. Chang and J.D. Yau, An element for analyzing vehicle-bridge systems considering vehicle's pitching effect, International Journal for Numerical Methods in Engineering 46(7) (1999), 1031-1047.

[16] Y.B. Yang and B.H. Lin, Vehicle-bridge interaction analysis by dynamic condensation method, Journal of Structural Engineering, ASCE 121(11) (1995), 1636-1643. 

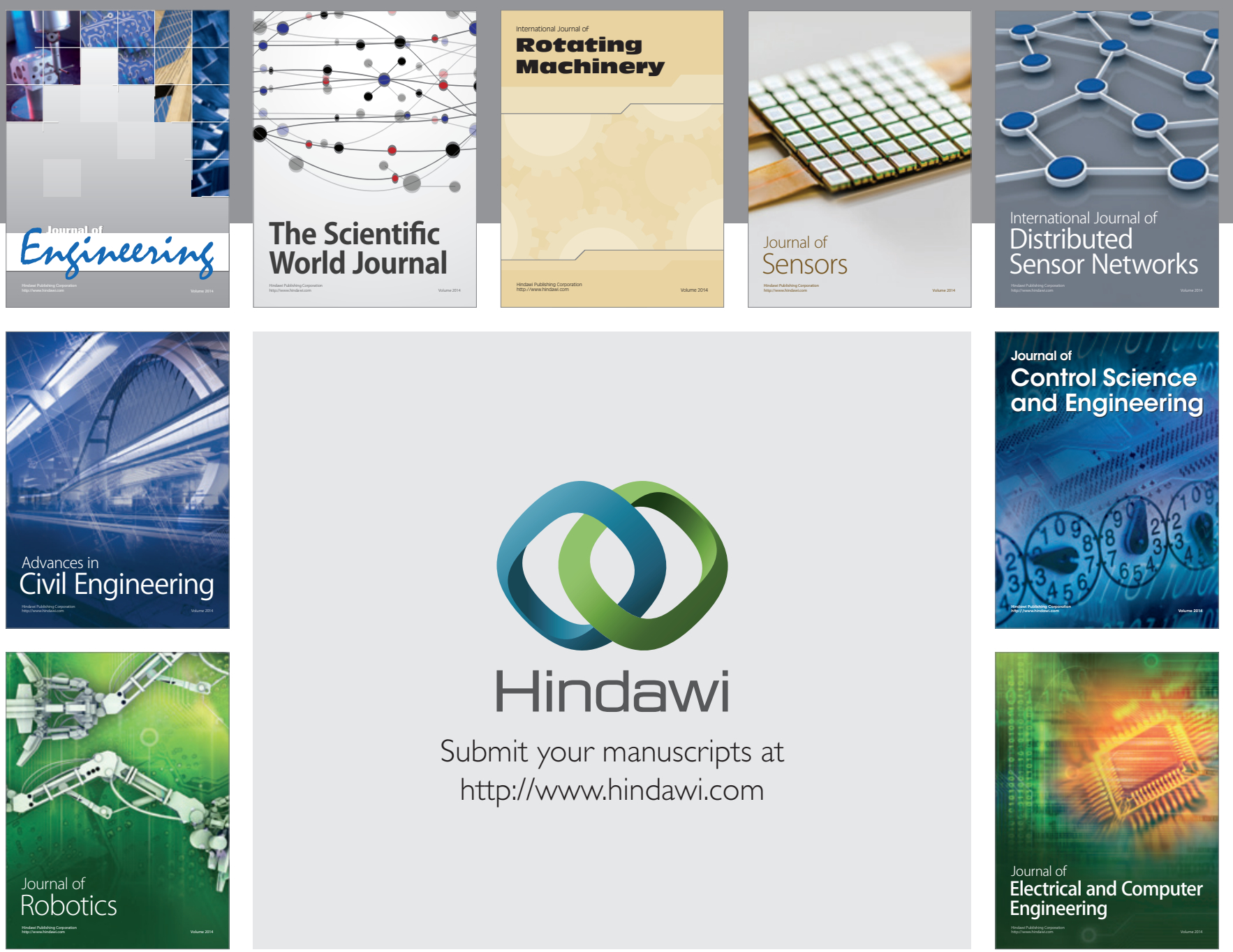

Submit your manuscripts at

http://www.hindawi.com
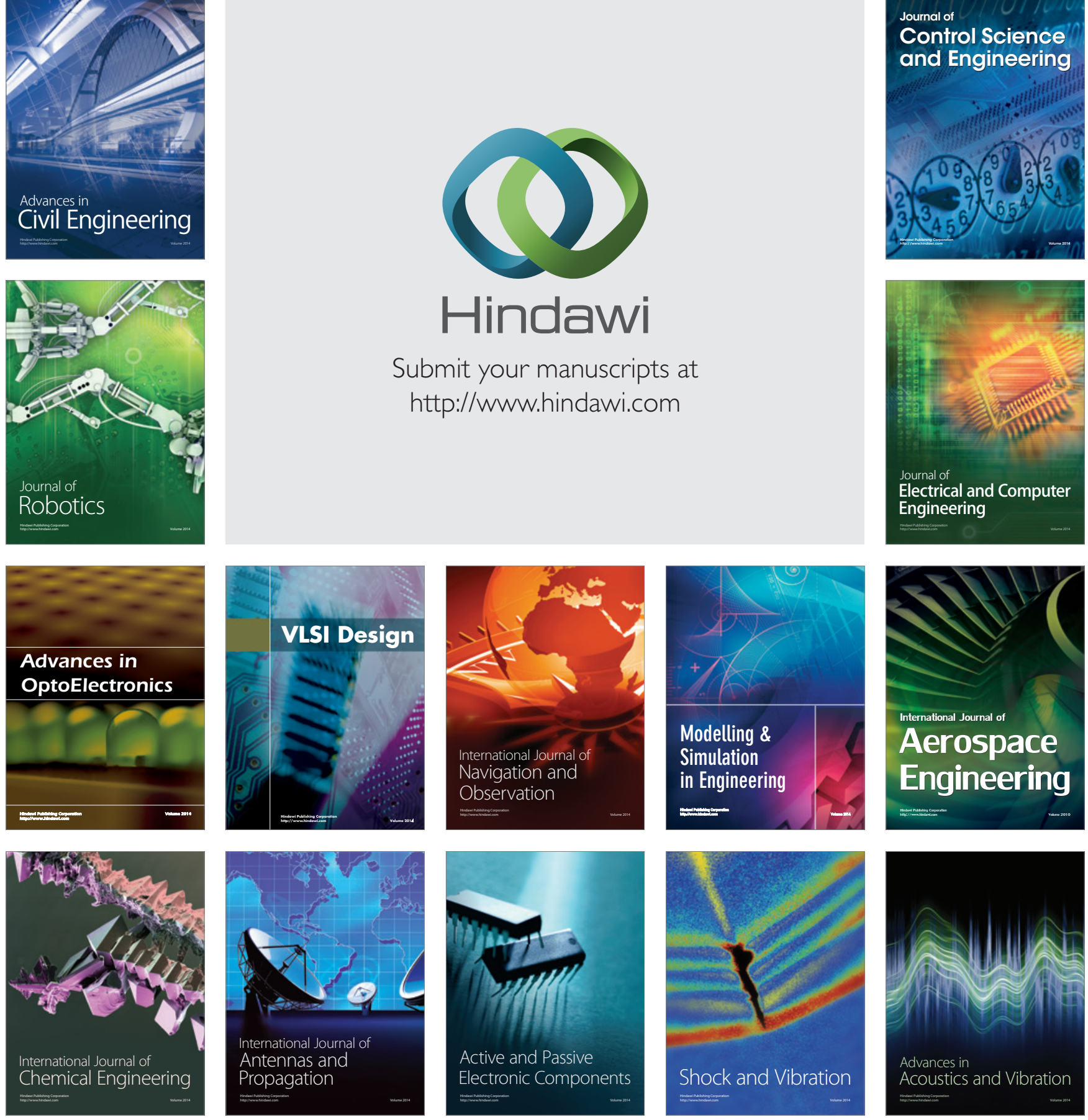